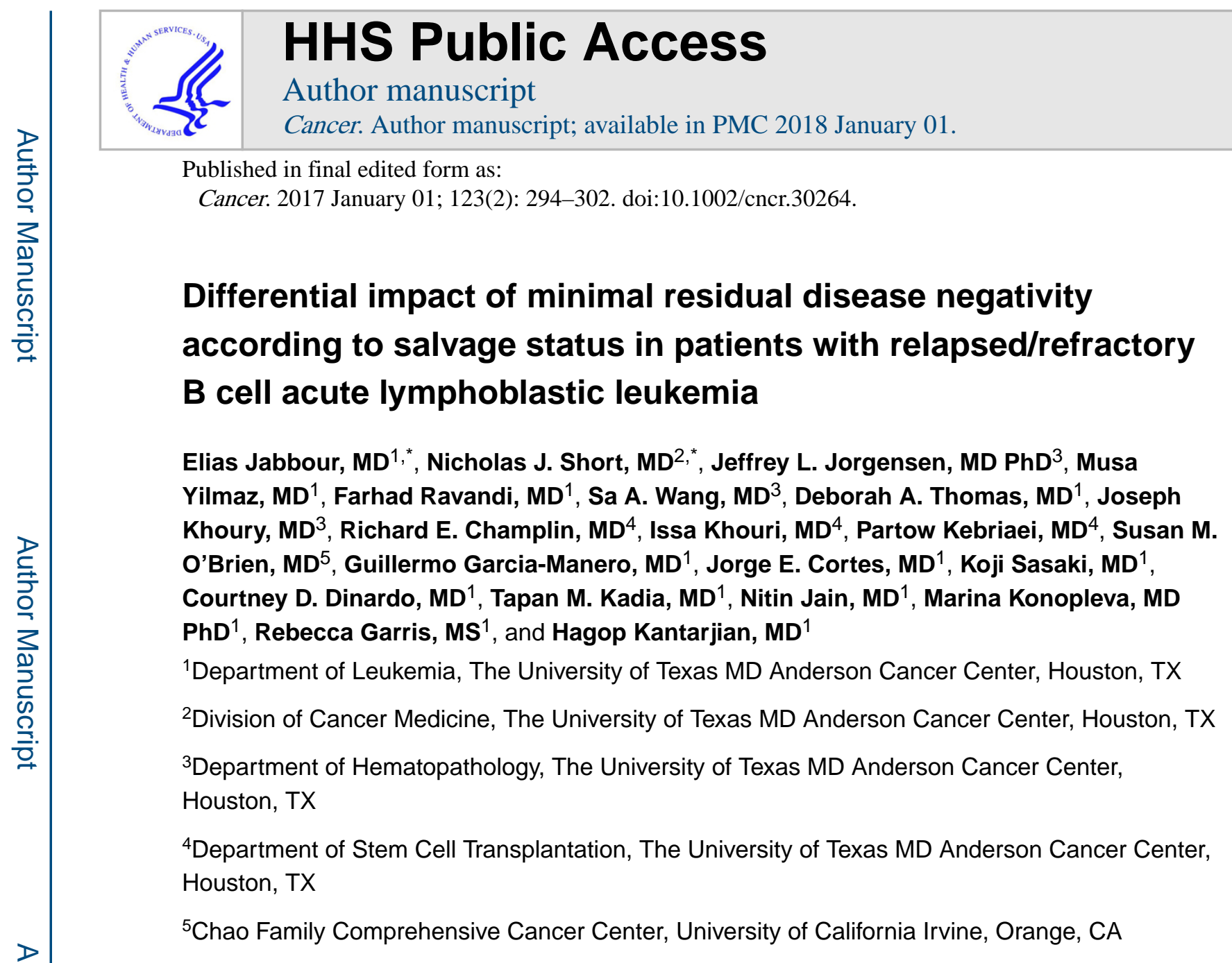

\title{
Abstract
}

Background-Minimal residual disease (MRD) assessment predicts survival in newly diagnosed acute lymphoblastic leukemia (ALL). Its significance in relapsed/refractory ALL is less clear.

Methods-We identified 78 patients with relapsed/refractory B-ALL who achieved morphologic response with inotuzumab ozogamicin $(n=41)$, blinatumomab $(n=11)$ or mini-hyper-CVD plus inotuzumab (HCVD+ino; $n=26)$ given in either salvage $1(\mathrm{~S} 1 ; \mathrm{n}=46)$ or salvage $2(\mathrm{~S} 2 ; \mathrm{n}=32)$ and who had MRD assessment by multiparameter flow cytometry at the time of remission.

Results-MRD negativity was achieved in 41 patients overall (53\%). The MRD negativity rate was $57 \%$ in S1 and 47\% in S2. Among patients in S1, achieving MRD negativity was associated with a longer EFS (median 18 months versus 7 months; 2-year EFS rate 46\% versus 17\%; $\mathrm{P}=0.06$ ) and OS (median 27 months versus 9 months; 2 -year OS 52\% versus 36\%; $\mathrm{P}=0.15$ ). EFS and OS were similar in S2 regardless of MRD response. Among MRD-negative patients who underwent

Correspondence: Elias Jabbour, MD, Department of Leukemia, Unit 428, The University of Texas MD Anderson Cancer Center, 1515 Holcombe Boulevard, Houston, TX 77030, USA; ejabbour@mdanderson.org.

E.J. and N.J.S. contributed equally to this study.

Authorship Contributions: N.J.S. and M.Y. designed the study, collected and analyzed the data, and wrote the manuscript; E.J. and H.K. designed the study, collected and analyzed the data, treated patients, and wrote the manuscript; K.S. designed the study and performed the statistical analysis; R.G. collected and analyzed the data; J.L.J., S.A.W., and J.K. assisted with pathologic interpretation; F.R., D.A.T., R.E.C, I.K., P.K., S.M.O., G.G-M., J.E.C., C.D.D., T.M.K, N.J., and M.K. treated patients. All authors reviewed and approved the manuscript. 
allogeneic stem cell transplant (SCT), EFS and OS were superior for those who underwent SCT in $\mathrm{S} 1$ rather than in $\mathrm{S} 2(\mathrm{P}=0.003$ and $\mathrm{P}=0.04$, respectively). Patients in $\mathrm{S} 1$ who achieved MRD negativity and subsequently underwent SCT had the best outcomes, with a 2-year OS rate of $65 \%$.

Conclusions-Patients with relapsed/refractory ALL who achieve MRD negativity in S1 can have long-term survival. Patients in S2 generally have poor outcomes regardless of MRD status.

\section{Condense Abstract}

In adults with relapsed/refractory ALL receiving first salvage therapy, achievement of MRD negativity is an important therapeutic outcome. Patients who achieve MRD negativity with first salvage treatment and undergo SCT have the best long-term survival.

\section{Keywords}

Acute lymphoblastic leukemia; minimal residual disease; relapsed; refractory; inotuzumab; blinatumomab

\section{Introduction}

In patients with newly diagnosed acute lymphoblastic leukemia (ALL), minimal residual disease (MRD) assessment after induction chemotherapy can help determine prognosis and risk-stratify patients for appropriate post-remission therapies. ${ }^{1-4}$ However, many patients still relapse, and their prognosis is dismal. ${ }^{5,6} \mathrm{New}$ monoclonal antibody therapies such as the anti-CD22 antibody-drug conjugate inotuzumab ozogamicin and the anti-CD19 bi-specific T-cell engager blinatumomab, either alone or in combination with cytotoxic chemotherapy, have recently shown promise for patients with relapsed or refractory ALL, improving outcomes compared to conventional chemotherapy, and with some patients experiencing long-term survival. ${ }^{7}$

Many studies have evaluated the prognostic role of MRD assessment in patients with newly diagnosed ALL. There are relatively few reports on the significance of MRD in patients with relapsed disease. These studies, which have primarily examined the prognostic significance of MRD in children in first relapse receiving cytotoxic chemotherapy, suggest that lower levels of MRD are associated with improved outcomes. ${ }^{8-11}$ In the pediatric population, lower levels of MRD in response to salvage treatment have also been associated with improved survival after allogeneic stem cell transplantation (SCT) ${ }^{12}$, and in one study, SCT appeared to decrease the risk for relapse or death in children who had an unsatisfactory MRD response to salvage therapy. ${ }^{13}$

In adults with ALL, the prognostic significance of MRD in relapsed/refractory ALL has been primarily reported in the context of individual studies using novel salvage treatments. Patients with relapsed/refractory ALL treated with blinatumomab and who achieved MRD negativity had longer survival than patients with remained MRD-positive. ${ }^{14,15}$ In one study of inotuzumab as salvage therapy, achievement of MRD negativity was associated with a longer remission duration. ${ }^{16}$ 
To clarify the prognostic impact of MRD assessment in adults with relapsed/refractory ALL, we performed a retrospective analysis of 78 patients treated at our institution who achieved remission with either first or second salvage therapy. Patients were treated with inotuzumab ozogamicin, blinatumomab, or mini-hyper-CVD (hyperfractionated cyclophosphamide, vincristine and doxorubicin) plus inotuzumab (HCVD+ino) and had MRD assessed at the time of response.

\section{Methods}

\section{Patients and Treatments}

Between June 2010 and May 2015, 130 adolescent and adult patients at our institution with relapsed/refractory B-ALL received treatment in either salvage $1(\mathrm{~S} 1 ; \mathrm{n}=68)$ or salvage ( $\mathrm{S} 2$; $\mathrm{n}=62$ ) with inotuzumab ozogamicin, blinatumomab or HCVD+ino. The inotuzumab and blinatumomab regimens have been previously published. ${ }^{14,16}$ The HCVD+ino regimen was delivered similarly to hyper-CVAD ${ }^{17}$ but used cyclophosphamide and dexamethasone at $50 \%$ dose reductions and omitted anthracyclines; on alternating cycles, methotrexate 250 $\mathrm{mg} / \mathrm{m}^{2}$ (75\% dose reduction) and cytarabine $0.5 \mathrm{~g} / \mathrm{m}^{2} \times 4$ doses ( $83 \%$ dose reduction) were given. Inotuzumab was administered on Day 3 of the first 4 cycles of therapy at a dose of $1.3-1.8 \mathrm{mg} / \mathrm{m}^{2}$ for the first cycle and $0.8-1.3 \mathrm{mg} / \mathrm{m}^{2}$ for subsequent cycles (depending on the time at which the patient was enrolled). Seventy-five patients (58\%) received inotuzumab, 35 (27\%) received HCVD+ino, and 20 (15\%) received blinatumomab (Figure 1). SCT was performed based on donor availability and at the discretion of the treating physician. Of the initial 130 patients, 78 (60\%) achieved morphological response with a median time to best response of 30 days (range, 13-99 days); these 78 patients are the subject of this analysis.

\section{Response Definitions}

A complete response (CR) was defined as the presence of $<5 \%$ blasts in the bone marrow (BM) aspirate, with $>1 \times 10^{9} / \mathrm{L}$ neutrophils and $\geq 100 \times 10^{9} / \mathrm{L}$ platelets in the peripheral blood, and no evidence of extramedullary disease. A CR with inadequate platelet recovery (CRp) was defined as meeting criteria for CR but with a platelet count $<100 \times 10^{9} / \mathrm{L}$. A CR with inadequate count recovery (CRi) was defined as $<5 \%$ blasts in the bone marrow aspirate and no evidence of extramedullary disease but not meeting criteria for either CR or CRp. Relapse was defined by recurrence of $25 \%$ blasts in a BM aspirate, or the presence of extramedullary disease. Event-free survival (EFS) was calculated from the time of treatment initiation until treatment failure, relapse or death from any cause. OS was calculated from the time of treatment initiation until death from any cause. Survival estimates were not censored at the time of SCT.

\section{Minimal Residual Disease}

MRD by multiparameter flow cytometry (MFC) was performed on remission BM specimens at the time of achievement of $\mathrm{CR} / \mathrm{CRp} / \mathrm{CRi}$. Initial MRD assessment was performed at approximately 4 weeks and then at least every 2 cycles of therapy. The MRD assay used a 6color panel as previously described. ${ }^{18}$ For patients treated with inotuzumab, the initial gate was set on CD19+ cells with low side-scatter light (SSC). In all cases the leukemic blasts 
had aberrancies in markers other than CD22, and CD22 expression was not included in the analysis for MRD. For patients treated with blinatumomab, the initial gate was set on CD10+ cells with low SSC. MRD positivity was defined on MFC scatter plots as a cluster of at least 20 cells showing altered expression of $\geq 2$ antigens. The sensitivity of this MRD assay is $₫ 0.01 \%$.

\section{Statistical Methods}

Patient characteristics were summarized using median (range) for continuous variables and frequencies (percentages) for categorical variables. Associations between categorical and continuous variables were assessed using chi-square tests. EFS and OS were calculated using Kaplan-Meier estimates; EFS and OS estimates were compared using the log-rank test. To evaluate the impact of SCT on survival outcomes, a 2-month landmark analysis was performed in which patients who relapsed or died within 2 months of achieving remission were excluded. By excluding patients with early death or relapse, this method allowed for more accurate determination of the potential impact of SCT on outcomes.

\section{Results \\ Baseline Characteristics}

Baseline characteristics of the study group are shown in Table 1 . The median age was 38 years (range, 18-87 years). Of the 78 patients who achieved remission with salvage therapy, 46 (59\%) were in S1 and 32 (41\%) were in S2. Forty-one patients (53\%) received inotuzumab, 11 (14\%) received blinatumomab, and 26 (33\%) received HCVD+ino. Twenty patients $(26 \%)$ had a normal karyotype at the time of salvage treatment, $4(5 \%)$ had $\mathrm{t}(9 ; 22)$, and $7(9 \%)$ had $\mathrm{t}(4 ; 11)$. Forty-eight patients $(36 \%)$ were refractory to previous therapy and 11 (14\%) had undergone prior SCT. Compared to patients in S1, patients in S2 had lower platelets $(\mathrm{P}=0.03)$, were more likely to have been refractory to last therapy $(\mathrm{P}<0.001)$ and were less likely to receive HCVD+ino $(\mathrm{P}=0.006)$.

Forty-eight patients (62\%) received hyper-CVAD-based chemotherapy as their initial treatment, 11 (14\%) received a Berlin-Frankfurt-Münster (BFM)-based regimen, and 19 (24\%) received other multiagent chemotherapy regimens. Among the patients in S2, previous salvage regimens included: multiagent chemotherapy in $21(66 \%)$, blinatumomab in $2(6 \%)$, CAR T-cells in $1(3 \%)$, moxetumomab pasudotox in $1(3 \%)$, ponatinib in $1(3 \%)$, and other investigational agents in $6(19 \%)$. No patients in the study population received prior inotuzumab, either alone or in combination with other agents.

\section{MRD Response by Treatment, Disease Status and Line of Therapy}

The median number of cycles to best response was 1 (range, 1-3). Fifty-two patients (67\%) achieved best response after 1 cycle of salvage therapy. MRD negativity was achieved in 41 patients (53\%). Rates of MRD negativity were similar among patients who achieved best response after 1 cycle compared to $\geq 2$ cycles ( $52 \%$ vs. 54\%, respectively). MRD negativity rates for patients in CR, CRp, and CRi were 57\%, 53\%, and 16\%, respectively. Among patients who achieved remission, MRD negativity was noted in 17 patients $(41 \%)$ treated with inotuzumab, 8 (73\%) treated with blinatumomab, and $16(62 \%)$ treated with HCVD 
+ino $(\mathrm{P}=0.10)$. Eight out of 11 patients $(72 \%)$ with prior SCT achieved MRD negativity. MRD negativity rates were similar for patients with refractory versus relapsed disease (54\% versus 51\%, respectively; $\mathrm{P}=0.83$ ). Twenty-six patients (57\%) in $\mathrm{S} 1$ and $15(47 \%)$ in $\mathrm{S} 2$ became MRD-negative $(\mathrm{P}=0.40)$. Among the 46 patients in $\mathrm{S} 1$, MRD negativity was achieved in 9 patients (22\%) who received inotuzumab, 3 (75\%) who received blinatumomab, and 14 (67\%) who received HCVD+ino. Among the 32 patients in S2, MRD negativity was achieved in $8(40 \%), 5(71 \%)$, and $2(40 \%)$, respectively. Four patients with Philadelphia chromosome-positive ALL were evaluated for MRD, 3 of whom achieved MRD negativity by MFC. Two of these 3 MRD-negative patients also had concomitant $B C R-A B L 1$ testing by polymerase chain reaction with detectable $B C R-A B L 1 / A B L 1$ ratios of $0.09 \%$ and $0.27 \%$.

\section{Survival Outcomes by MRD Response}

With a median follow-up duration of 27 months (range, 6-55 months), 38 (48\%) of the 78 evaluable patients have relapsed. Of the patients who relapsed, 21 (51\%) were MRDnegative and 17 (46\%) were MRD-positive after salvage treatment. The median EFS was 12 months in patients who were MRD-negative and 6 months for those who were MRDpositive; the 2-year EFS rates were $31 \%$ and $12 \%$, respectively ( $\mathrm{P}=0.09$; Figure $2 \mathrm{~A}$ ). Fiftyone patients (65\%) have died, 26 (61\%) of those who achieved MRD negativity and 27 (73\%) who remained MRD-positive. The median OS was 17 months for patients who were MRD-negative and 9 months for those who were MRD-positive; the 2-year OS rates were $40 \%$ and $26 \%$, respectively $(\mathrm{P}=0.18$; Figure $2 \mathrm{~B})$.

\section{Impact of Salvage Status on Outcomes}

When patients were stratified according to salvage status, a differential impact on survival was observed (Figure 3). As expected, for patients who achieved MRD negativity, those in $\mathrm{S} 1$ had significantly longer EFS (median 18 months vs. 5 months; $\mathrm{P}=0.001$ ) and $\mathrm{OS}$ (median 27 months vs. 7 months; $\mathrm{P}=0.01$ ) compared to those in $\mathrm{S} 2$ (Figure 3A). In contrast, for patients who remained MRD positive, EFS and OS were similar regardless of salvage status ( $\mathrm{P}=0.41$, and $\mathrm{P}=0.39$, respectively).

Among patients in S1, those who achieved MRD negativity had longer EFS (median 18 months vs. 7 months; 2-year EFS rate 46\% vs. 17\%; $\mathrm{P}=0.06$ ) and OS (median 27 months vs. 9 months; 2-year OS 52\% vs. $36 \%$; $\mathrm{P}=0.15$ ) compared to those who were MRD-positive (Figure 3B). In contrast, for patients in S2, EFS and OS were similar among MRD-negative and MRD-positive patients $(\mathrm{P}=0.88$, and $\mathrm{P}=0.99$, respectively).

Among 11 patients with prior SCT, long-term survival was observed in a subgroup of patients who achieved MRD negativity. All 3 patients with prior SCT who remained MRDpositive died with a median OS of 8 months, compared to a median OS of 39 months for the 8 patients with prior SCT who achieved MRD negativity $(\mathrm{P}=0.04)$. The median EFS was also longer in the MRD-negative group compared to the MRD-positive group (18 months vs. 8 months; $\mathrm{P}=0.01$ ). Four of the $8 \mathrm{MRD}$-negative patients are still alive, 2 of whom subsequently underwent SCT. The median survival duration of these patients was 29 months (range, 27-55 months). 


\section{Impact of SCT and MRD Status on Outcomes}

Fourteen patients (18\%) relapsed or died within 2 months of achieving remission and were excluded for purposes of the landmark analysis. Of the 64 remaining patients, 40 (63\%) were in S1 and 24 (38\%) were in S2. Overall, 42 of these patients (66\%) underwent SCT with a median time to SCT of 2 months, 20 of whom had achieved MRD negativity and 22 of whom had remained MRD positive at the time of response to salvage treatment. The rates of SCT were similar among MRD-negative and MRD positive patients (63\% and 69\%, respectively) and among those in S1 and S2 (65\% and 67\%, respectively).

Among the 64 patients included in the landmark analysis, 13 are still alive with $>2$ years of follow-up. Long-term survival was observed in all groups of patients regardless of salvage treatment (inotuzumab: $\mathrm{n}=6$; blinatumomab: $\mathrm{n}=3$; HCVD+ino: $\mathrm{n}=4$ ), salvage status ( $\mathrm{S} 1$ : $\mathrm{n}=10 ; \mathrm{S} 2, \mathrm{n}=3$ ) or MRD status (negative: $\mathrm{n}=9$; positive: $\mathrm{n}=4)$. Of these 13 patients, $8(62 \%)$ had undergone SCT.

Overall, EFS and OS did not differ between patients who did or did not undergo SCT $(\mathrm{P}=0.84$ and $\mathrm{P}=0.97$, respectively). Nevertheless, a clear trend for improved long-term outcomes with SCT was seen and small numbers may account for the lack of significance. Among patients who achieved MRD negativity, the median EFS was 17 months and 12 months, and 2-year EFS rates were $46 \%$ and $28 \%$ for patients who underwent SCT compared to those who did not $(\mathrm{P}=0.24)$. The median OS was 24 months and 23 months, and 2-year OS rates were 55\% and 46\%, respectively ( $\mathrm{P}=0.41)$. For MRD-positive patients, the median EFS was 6 months and 9 months, and 2-year EFS rates were $11 \%$ and 25\%, respectively ( $\mathrm{P}=0.48)$. Similarly, among MRD-positive patients, the median OS was 10 months and 9 months, and 2-year OS rates were $23 \%$ and $36 \%$, respectively $(\mathrm{P}=0.56)$. Among patients who underwent SCT, those who were MRD-negative at response had significantly longer EFS and OS compared to those who were MRD-positive ( $\mathrm{P}=0.006$ and $\mathrm{P}=0.02$, respectively).

\section{Effect of Salvage Status on Post-SCT Outcomes}

When stratified according to salvage status, patients who achieved MRD negativity after S1 treatment and subsequently underwent SCT appeared to have best outcomes (Figure 4). Of the 22 patients who achieved MRD negativity after S1 treatment, the median EFS for patients who underwent SCT $(n=14)$ compared to those who did not $(n=8)$ was not reached vs. 18 months, and the median OS was not reached vs. 27 months, respectively ( $\mathrm{P}=0.14$ and $\mathrm{P}=0.28$, respectively). EFS and OS were similar among MRD-positive patients, regardless of whether SCT was performed ( $\mathrm{P}=0.51$ and $\mathrm{P}=0.53$, respectively). Among the 14 patients who achieved MRD negativity after S1 treatment and subsequently underwent SCT, 10 (71\%) are still alive with a median follow-up of 24 months (range, 5-55 months). Of the 4 patients who died, 2 died from relapsed ALL and 2 from complications of graft-versus-host disease.

In contrast, EFS and OS were similar among patients in S2 regardless of MRD status or SCT (Figure 5). Among MRD-negative patients who underwent SCT, EFS and OS were superior for those who underwent SCT in $\mathrm{S} 1$ rather than in $\mathrm{S} 2(\mathrm{P}=0.003$ and $\mathrm{P}=0.04$, respectively). 
However, EFS and OS were similar among MRD-positive patients who underwent SCT, regardless of salvage status $(\mathrm{P}=0.54$ and $\mathrm{P}=0.33$, respectively).

\section{Discussion}

The achievement of MRD negativity in response to frontline chemotherapy is predictive for improved survival among patients with ALL, both in the pediatric and adult populations. ${ }^{1,2,4,19}$ In this study of patients with relapsed/refractory B-ALL, we found achievement of MRD negativity to be associated with improved outcomes even in the salvage setting, resulting in an approximately 2-fold increase in both EFS and OS, compared to those patients who remained MRD-positive. Achievement of MRD negativity was seen across treatments and with similar frequencies regardless of salvage status or whether the patient had relapsed or was refractory to previous treatment. However, the beneficial prognostic impact of achieving MRD negativity was limited to patients in S1. In particular, we identified a subgroup of patients who achieved MRD negativity in S1 and subsequently received SCT that had excellent long-term outcomes. These findings suggest that MRD assessment plays an important prognostic role in the relapsed/refractory setting, although its utility is strongly influenced by salvage status.

Despite similar rates of MRD negativity among those in S1 and in S2 (57\% versus 47\%, respectively), MRD status was prognostic only in patients in S1. Among patients in S1, achievement of MRD negativity was associated with an increase in median OS and a favorable 2-year OS rate of 52\%. In contrast, outcomes were similar among patients in S2, regardless of MRD status at remission. Notably, those patients who achieved MRD negativity with S1 and subsequently underwent SCT had the best outcome, with $71 \%$ of patients still alive after a median follow-up of 2 years. The survival of patients who achieved MRD negativity in S1 (median OS: 27 months) compares very favorably to the historical median OS of 4-5 months reported in adult patients with refractory or first relapsed ALL treated with standard chemotherapy-based salvage regimens. ${ }^{5,6}$ Taken together, these findings suggest that patients with relapsed/refractory ALL who achieve MRD negativity in S1 can have excellent long-term outcomes, especially if salvage SCT is performed.

The finding that achievement of MRD negativity is an important therapeutic endpoint in S1 but not in S2 raises several intriguing questions. The lack of prognostic significance of MRD negativity by MFC in S2 highlights the need for more sensitive assays to detect MRD. Nextgeneration sequencing holds promise in identifying MRD with a higher level of sensitivity than MFC, although experience with this approach is relatively limited. ${ }^{20,21}$ Additionally, next-generation sequencing has the ability to detect subclonal disease that might predispose to relapse but which might be missed by MFC, either due to lack of sensitivity of the flow cytometry assay or due to immunophenotypic shifting of the relapsing clone. ${ }^{22}$ Unlike in acute myeloid leukemia in which clonal heterogeneity and clonal evolution leading to relapse is well-established ${ }^{23,24}$, less is known about subclonality in adult ALL. It is possible patients with exposure to more prior therapies have an increased likelihood of resistant subclones that persist below the level of detection of the MFC-based MRD assay. Future studies evaluating the clonal heterogeneity of relapsed/refractory ALL as well as the 
mechanisms of relapse are warranted and may provide insight into our finding of the poor predictive value of MFC in the S2 population.

Ultimately, regardless of the reason for the differential prognostic impact of MRD according to salvage status, our findings strongly support the strategy of using the most effective available therapy (e.g. blinatumomab and inotuzumab) at the time of first relapse, followed by SCT for responding patients with an adequate donor. These data suggest that the same depth of response as assessed by MFC (i.e. MRD negativity) leads to divergent survival outcomes in these two groups of patients. For patients in S2, novel treatment strategies for these patients are needed, as this group has a particularly poor prognosis regardless of MRD response..$^{25}$

In this study we analyzed patients treated with one of three monoclonal antibody-containing regimens: inotuzumab, blinatumomab and HCVD+ino. Inotuzumab and blinatumomab have shown significant promise in the management of relapsed/refractory ALL and appear to improve outcomes compared to historical data using chemotherapy-only regimens. ${ }^{7,26}$ The apparent improved survival observed with these novel monoclonal antibodies may be in part mediated through the higher MRD negativity rates achieved with these regimens as compared to standard cytotoxic chemotherapy. ${ }^{27}$ For example, in a long-term follow-up of patients who received blinatumomab in the salvage setting, MRD response was associated with a $67 \%$ reduction in the risk of death. ${ }^{15}$ In studies of patients who remained MRDpositive after initial treatment or who developed MRD relapse, blinatumomab has been shown to be particularly effective in eliminating MRD, which appears to translate to improved survival among MRD responders. ${ }^{28}$ Our findings suggest that MRD response in the salvage setting may translate into improved outcomes among patients treated with novel monoclonal antibody-based regimens. However, whether MRD-directed therapies for patients who remain MRD-positive after salvage treatment will also improve outcomes needs to be evaluated in prospective studies.

Our study has several limitations. Although we identified strong trends towards improved survival for patients who achieved MRD negativity (especially those in S1), these analyses did not reach statistical significance, likely due to the relatively small sample size.

Furthermore, we were not able to assess the optimal timing of MRD assessment in the salvage setting, as MRD was only measured at the time of remission. In the frontline setting, the prognostic impact of MRD response varies based on the timing of MRD assessment, and therefore future studies to evaluate this issue in patients with relapsed/refractory disease are warranted. ${ }^{29}$ Finally, the finding that relapses were still frequent among patients who achieved MRD negativity highlights the importance of developing newer, more sensitive assays for MRD.

In conclusion, patients with relapsed/refractory ALL who achieve MRD negativity in S1 have improved outcomes and can achieve excellent long-term survival, especially if SCT is performed. Achievement of MRD negativity is an important therapeutic outcome in the relapsed/refractory setting but has differential prognostic impact based on salvage status. 


\section{Acknowledgments}

Funding source: Supported by the MD Anderson Cancer Center Support Grant CA016672 and the "Charif Souki Cancer Research Fund" P30 CA016672, Ronald DePinho

Disclosure of Conflicts of Interest: E.J. and H.K. received research grants from Pfizer and Amgen.

\section{References}

1. Bruggemann M, Raff T, Flohr T, et al. Clinical significance of minimal residual disease quantification in adult patients with standard-risk acute lymphoblastic leukemia. Blood. 2006; 107(3):1116-1123. [PubMed: 16195338]

2. Patel B, Rai L, Buck G, et al. Minimal residual disease is a significant predictor of treatment failure in non T-lineage adult acute lymphoblastic leukaemia: final results of the international trial UKALL XII/ECOG2993. Br J Haematol. 2010; 148(1):80-89. [PubMed: 19863538]

3. Ribera JM, Oriol A, Morgades M, et al. Treatment of high-risk Philadelphia chromosome-negative acute lymphoblastic leukemia in adolescents and adults according to early cytologic response and minimal residual disease after consolidation assessed by flow cytometry: final results of the PETHEMA ALL-AR-03 trial. J Clin Oncol. 2014; 32(15):1595-1604. [PubMed: 24752047]

4. Ravandi F, Jorgensen JL, O'Brien SM, et al. Minimal residual disease assessed by multi-parameter flow cytometry is highly prognostic in adult patients with acute lymphoblastic leukaemia. $\mathrm{Br} \mathbf{J}$ Haematol. 2016; 172(3):392-400. [PubMed: 26492205]

5. Oriol A, Vives S, Hernandez-Rivas JM, et al. Outcome after relapse of acute lymphoblastic leukemia in adult patients included in four consecutive risk-adapted trials by the PETHEMA Study Group. Haematologica. 2010; 95(4):589-596. [PubMed: 20145276]

6. Kantarjian HM, Thomas D, Ravandi F, et al. Defining the course and prognosis of adults with acute lymphocytic leukemia in first salvage after induction failure or short first remission duration. Cancer. 2010; 116(24):5568-5574. [PubMed: 20737576]

7. Jabbour E, O'Brien S, Ravandi F, Kantarjian H. Monoclonal antibodies in acute lymphoblastic leukemia. Blood. 2015; 125(26):4010-4016. [PubMed: 25999456]

8. Eckert C, Biondi A, Seeger K, et al. Prognostic value of minimal residual disease in relapsed childhood acute lymphoblastic leukaemia. Lancet. 2001; 358(9289):1239-1241. [PubMed: 11675066]

9. Coustan-Smith E, Gajjar A, Hijiya N, et al. Clinical significance of minimal residual disease in childhood acute lymphoblastic leukemia after first relapse. Leukemia. 2004; 18(3):499-504. [PubMed: 14981525]

10. Paganin M, Zecca M, Fabbri G, et al. Minimal residual disease is an important predictive factor of outcome in children with relapsed 'high-risk' acute lymphoblastic leukemia. Leukemia. 2008; 22(12):2193-2200. [PubMed: 18754029]

11. Eckert C, von Stackelberg A, Seeger K, et al. Minimal residual disease after induction is the strongest predictor of prognosis in intermediate risk relapsed acute lymphoblastic leukaemia long-term results of trial ALL-REZ BFM P95/96. Eur J Cancer. 2013; 49(6):1346-1355. [PubMed: 23265714]

12. Bader P, Kreyenberg H, Henze GH, et al. Prognostic value of minimal residual disease quantification before allogeneic stem-cell transplantation in relapsed childhood acute lymphoblastic leukemia: the ALL-REZ BFM Study Group. J Clin Oncol. 2009; 27(3):377-384. [PubMed: 19064980]

13. Eckert C, Henze G, Seeger K, et al. Use of allogeneic hematopoietic stem-cell transplantation based on minimal residual disease response improves outcomes for children with relapsed acute lymphoblastic leukemia in the intermediate-risk group. J Clin Oncol. 2013; 31(21):2736-2742. [PubMed: 23775972]

14. Topp MS, Gokbuget N, Stein AS, et al. Safety and activity of blinatumomab for adult patients with relapsed or refractory B-precursor acute lymphoblastic leukaemia: a multicentre, single-arm, phase 2 study. Lancet Oncol. 2015; 16(1):57-66. [PubMed: 25524800] 
15. Zugmaier G, Gokbuget N, Klinger M, et al. Long-term survival and T-cell kinetics in relapsed/ refractory ALL patients who achieved MRD response after blinatumomab treatment. Blood. 2015; 126(24):2578-2584. [PubMed: 26480933]

16. Kantarjian H, Thomas D, Jorgensen J, et al. Results of inotuzumab ozogamicin, a CD22 monoclonal antibody, in refractory and relapsed acute lymphocytic leukemia. Cancer. 2013; 119(15):2728-2736. [PubMed: 23633004]

17. Kantarjian HM, O'Brien S, Smith TL, et al. Results of treatment with hyper-CVAD, a doseintensive regimen, in adult acute lymphocytic leukemia. J Clin Oncol. 2000; 18(3):547-561. [PubMed: 10653870]

18. Ravandi F, Jorgensen JL, Thomas DA, et al. Detection of MRD may predict the outcome of patients with Philadelphia chromosome-positive ALL treated with tyrosine kinase inhibitors plus chemotherapy. Blood. 2013; 122(7):1214-1221. [PubMed: 23836561]

19. Gokbuget N, Kneba M, Raff T, et al. Adult patients with acute lymphoblastic leukemia and molecular failure display a poor prognosis and are candidates for stem cell transplantation and targeted therapies. Blood. 2012; 120(9):1868-1876. [PubMed: 22442346]

20. Pan X, Nariai N, Fukuhara N, et al. Monitoring of minimal residual disease in early T-cell precursor acute lymphoblastic leukaemia by next-generation sequencing. Br J Haematol. 2016

21. Kotrova M, Muzikova K, Mejstrikova E, et al. The predictive strength of next-generation sequencing MRD detection for relapse compared with current methods in childhood ALL. Blood. 2015; 126(8):1045-1047. [PubMed: 26294720]

22. van Dongen JJ, van der Velden VH, Bruggemann M, Orfao A. Minimal residual disease diagnostics in acute lymphoblastic leukemia: need for sensitive, fast, and standardized technologies. Blood. 2015; 125(26):3996-4009. [PubMed: 25999452]

23. Ding L, Ley TJ, Larson DE, et al. Clonal evolution in relapsed acute myeloid leukaemia revealed by whole-genome sequencing. Nature. 2012; 481(7382):506-510. [PubMed: 22237025]

24. Shlush LI, Zandi S, Mitchell A, et al. Identification of pre-leukaemic haematopoietic stem cells in acute leukaemia. Nature. 2014; 506(7488):328-333. [PubMed: 24522528]

25. Jabbour E, O'Brien S, Huang X, et al. Prognostic factors for outcome in patients with refractory and relapsed acute lymphocytic leukemia treated with inotuzumab ozogamicin, a CD22 monoclonal antibody. Am J Hematol. 2015; 90(3):193-196. [PubMed: 25407953]

26. Yilmaz M, Richard S, Jabbour E. The clinical potential of inotuzumab ozogamicin in relapsed and refractory acute lymphocytic leukemia. Ther Adv Hematol. 2015; 6(5):253-261. [PubMed: 26425338]

27. DeAngelo D, Stelljes M, Martinelli G, et al. Efficacy and safety of inotuzumab ozogamicin (INO) vs standard of care (SOC) in salvage 1 or 2 in patients with acute lymphoblastic leukemia (ALL): an ongoing global phase 3 study. Haematologica. 2015; 100 S1 abstract \#LB2073.

28. Gökbuget N, Dombret H, Bonifacio M, et al. Long-Term Outcomes after Blinatumomab Treatment: Follow-up of a Phase 2 Study in Patients (Pts) with Minimal Residual Disease (MRD) Positive B-Cell Precursor Acute Lymphoblastic Leukemia (ALL). Blood. 2015; 126(23):680-680.

29. Schrappe M. Minimal residual disease: optimal methods, timing, and clinical relevance for an individual patient. Hematology Am Soc Hematol Educ Program. 2012; 2012:137-142. [PubMed: 23233572] 
All Patients

$(\mathrm{N}=130)$
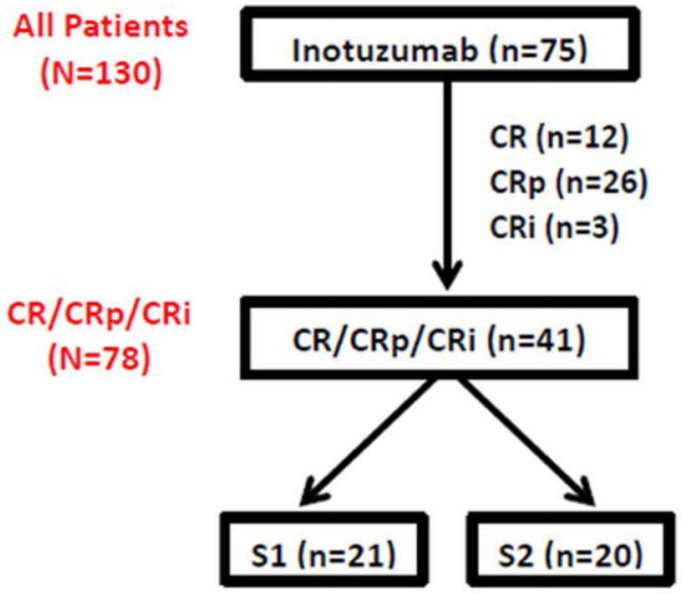

Figure 1. Study population
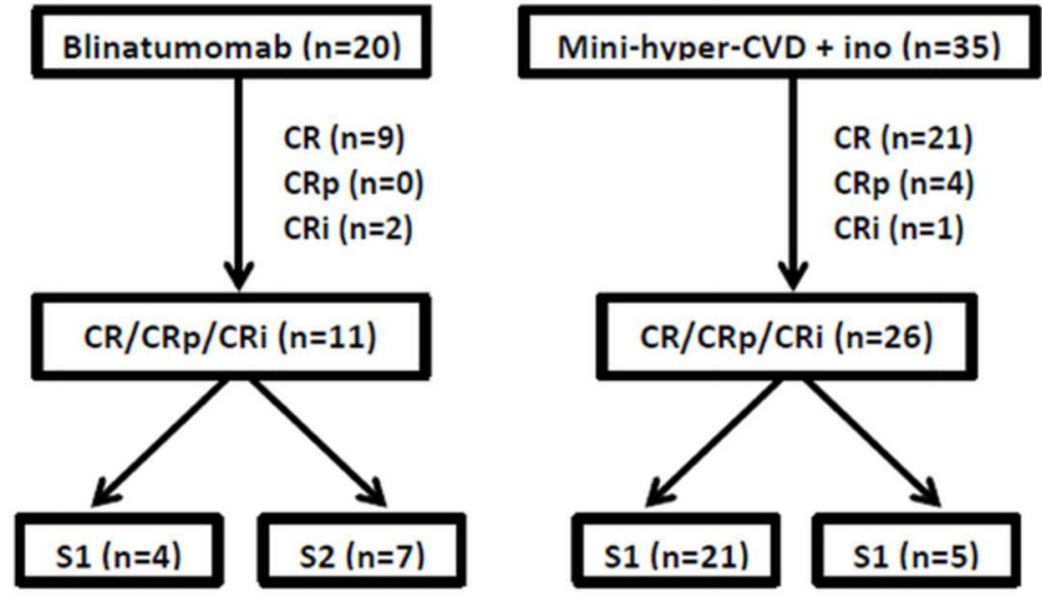

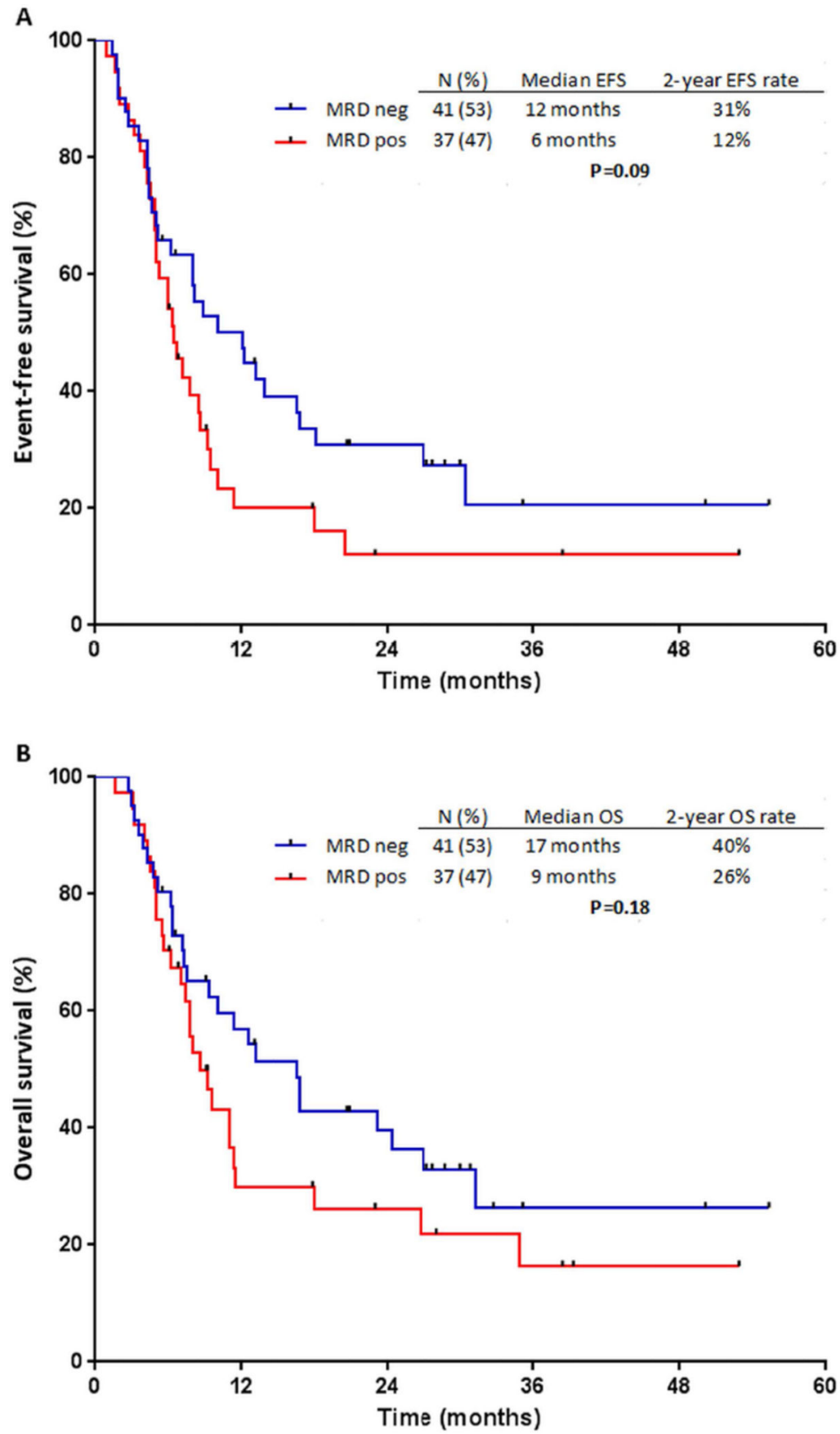

Figure 2. Outcomes by minimal residual disease (MRD) response

(A) Event-free survival (EFS) and (B) overall survival (OS). 

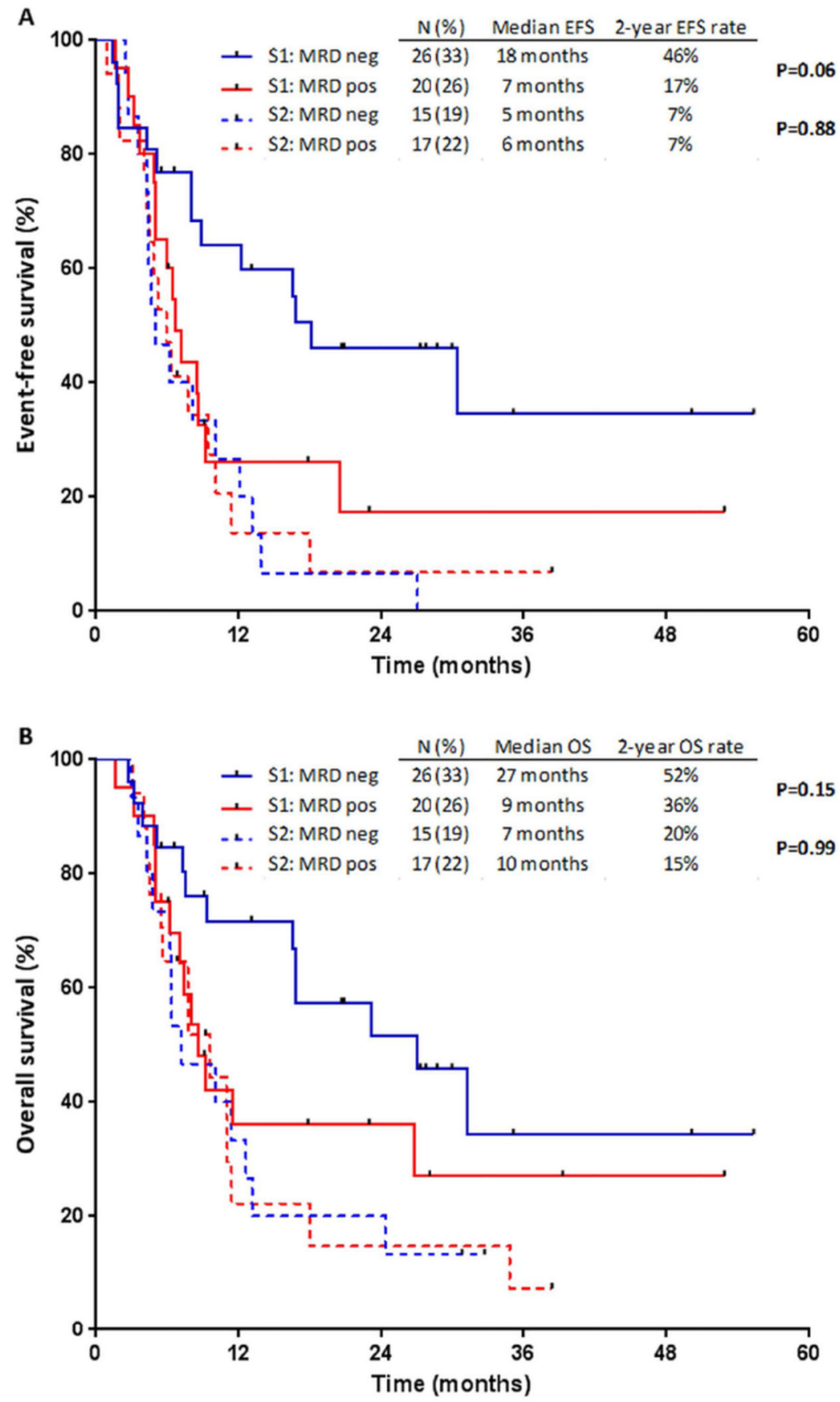

Figure 3. Outcomes by minimal residual disease (MRD) response, stratified by salvage status (A) Event-free survival (EFS) and (B) overall survival (OS). 

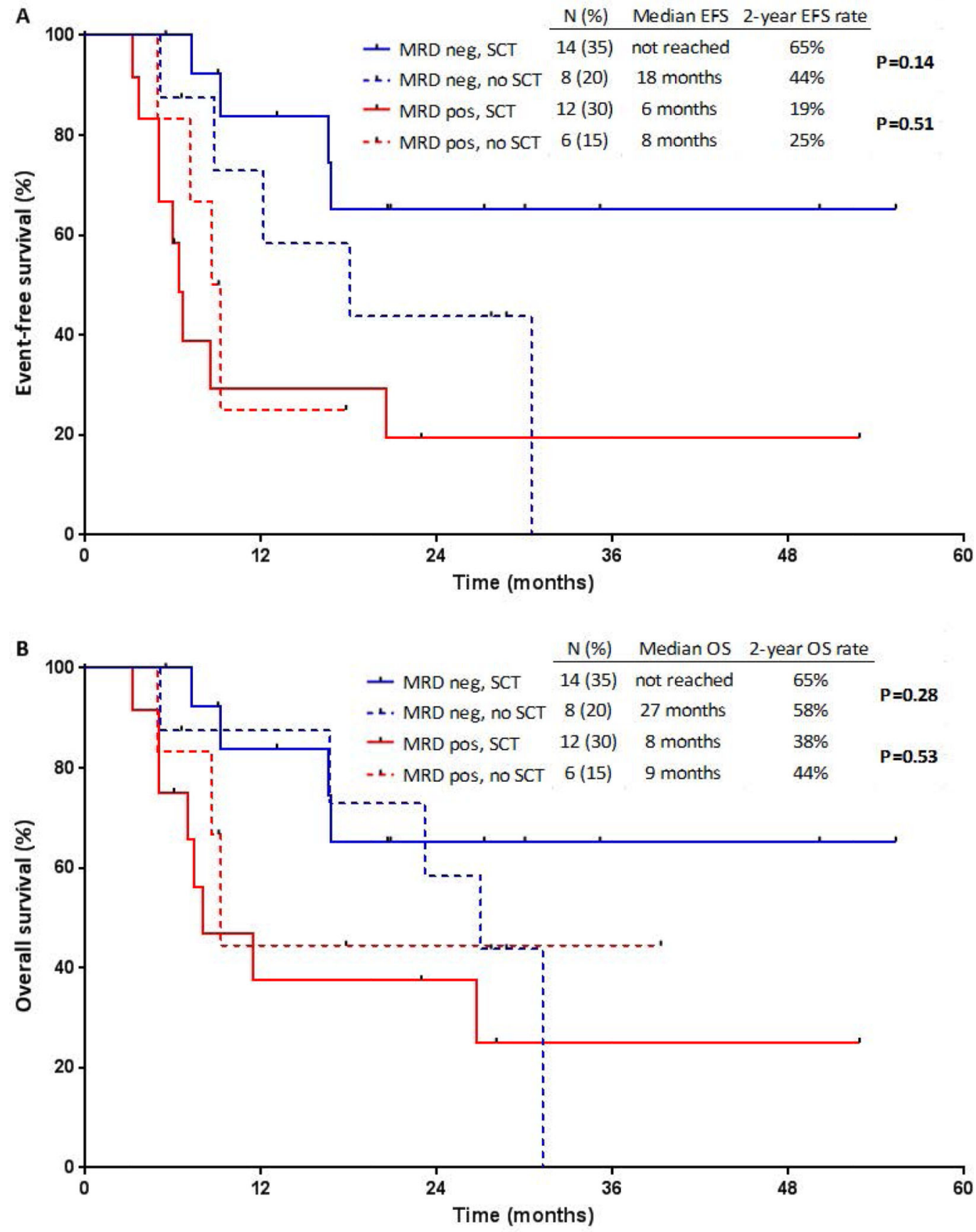

Figure 4. Outcomes of patients after first salvage treatment, stratified by minimal residual disease (MRD) status and stem cell transplantation (SCT)

(A) Event-free survival (EFS) and (B) overall survival (OS). 
A
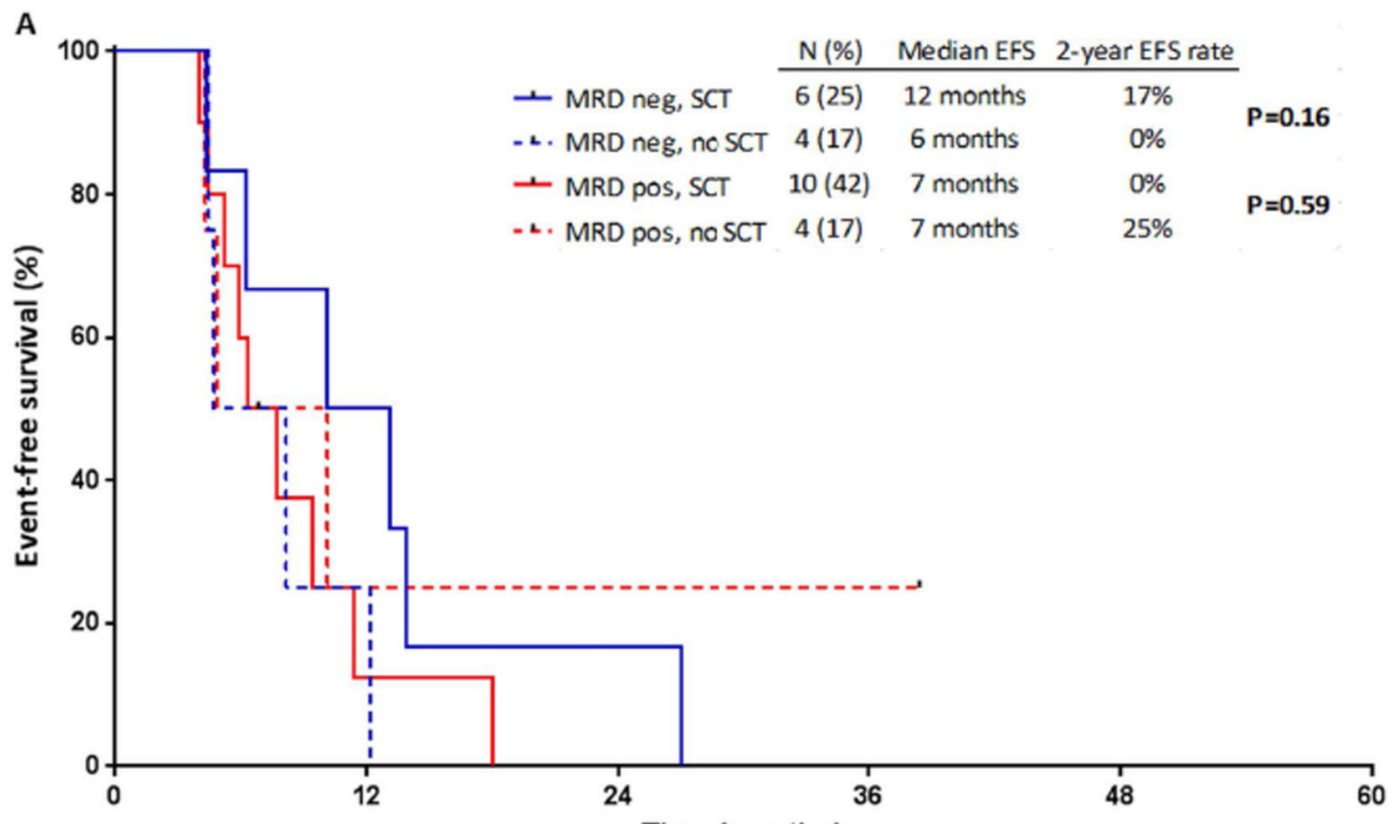

B

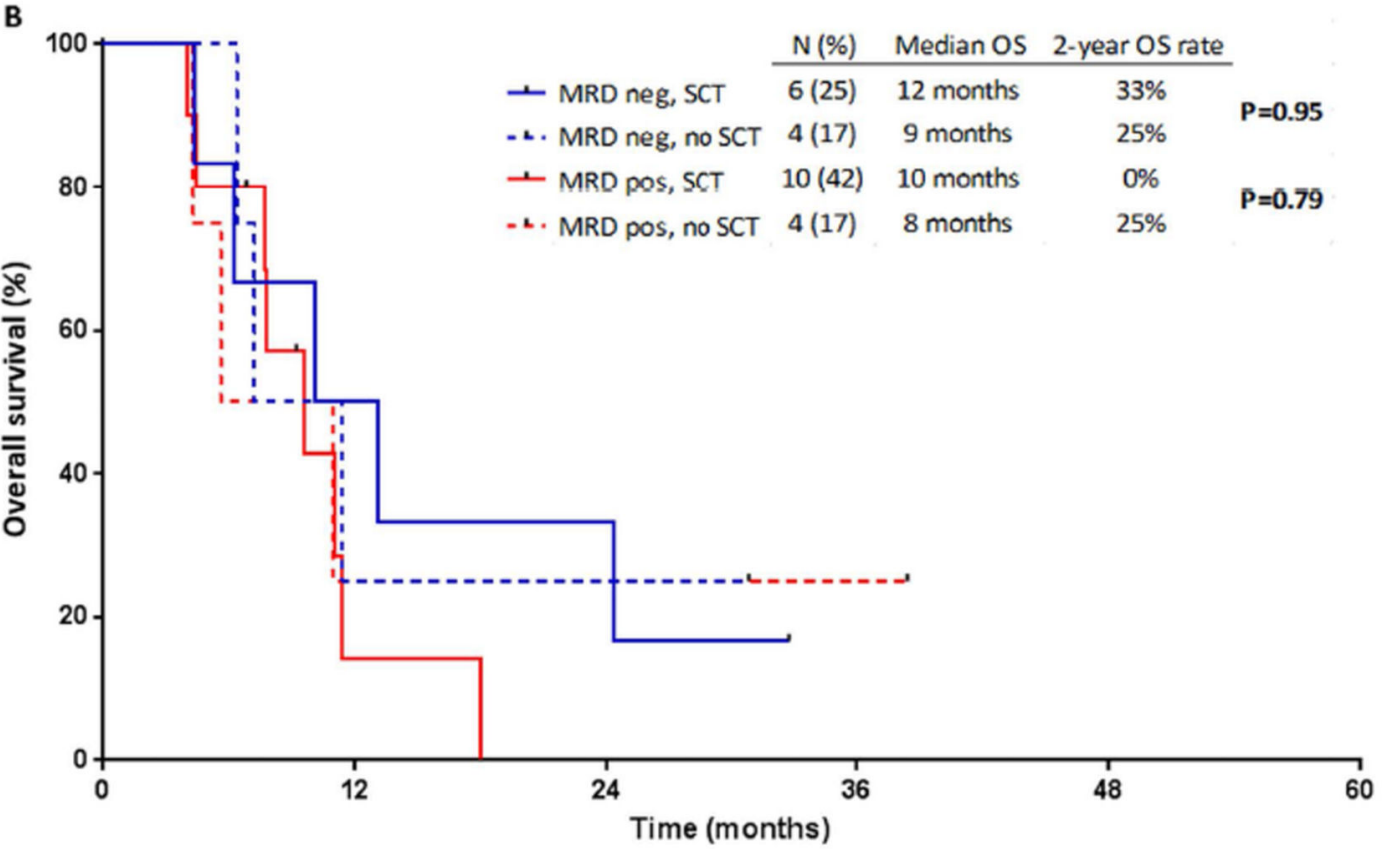

Figure 5. Outcomes of patients after second salvage treatment, stratified by minimal residual disease (MRD) status and stem cell transplantation (SCT)

(A) Event-free survival (EFS) and (B) overall survival (OS). 


\section{Table 1}

Baseline characteristics by salvage status

\begin{tabular}{|c|c|c|c|}
\hline Characteristic $^{a}$ & $\begin{array}{c}\text { Salvage } 1 \\
(n=46)\end{array}$ & $\underset{(n=32)}{\text { Salvage } 2}$ & $\begin{array}{l}\text { All patients } \\
(\mathbf{N}=78)\end{array}$ \\
\hline Age (years) & $38(18-87)$ & $38(19-79)$ & $38(18-87)$ \\
\hline WBC $\left(10^{9} / L\right)$ & $3.2(0.5-36.8)$ & $2.9(0.3-37.6)$ & $3.1(0.3-37.6)$ \\
\hline Hemoglobin (g/dL) & $10.0(7.6-15.9)$ & $10.3(7.7-14.4)$ & $10.1(7.6-15.9)$ \\
\hline Platelets $\left(10^{9} / \mathrm{L}\right)$ & $98(9-394)$ & $37(7-286)$ & $64(7-394)$ \\
\hline BM blasts (\%) & $64(8-97)$ & $51(10-96)$ & $61(8-97)$ \\
\hline LDH (U/L) & $551(231-9043)$ & $695(266-19165)$ & $585(231-19165)$ \\
\hline CNS disease & $2(4)$ & $0(0)$ & $2(3)$ \\
\hline \multicolumn{4}{|l|}{ Cytogenetics } \\
\hline Diploid & $13(28)$ & $6(19)$ & $19(24)$ \\
\hline$t(9 ; 22)$ & $2(4)$ & $2(6)$ & $4(5)$ \\
\hline$t(4 ; 11)$ & $6(13)$ & $1(2)$ & $7(9)$ \\
\hline Others & $25(54)$ & $23(72)$ & $48(62)$ \\
\hline \multicolumn{4}{|l|}{ Performance status } \\
\hline $0-1$ & $44(96)$ & $29(91)$ & $73(94)$ \\
\hline $2-4$ & $2(4)$ & $3(9)$ & $5(6)$ \\
\hline \multicolumn{4}{|l|}{ Disease status } \\
\hline Refractory & $9^{b}(20)$ & $19(59)$ & $28(36)$ \\
\hline Relapsed & $36(80)$ & $13(41)$ & $49(64)$ \\
\hline Prior SCT & $5(11)$ & $6(19)$ & $11(14)$ \\
\hline \multicolumn{4}{|l|}{ Salvage Treatment } \\
\hline Inotuzumab & $21(46)$ & $20(63)$ & $41(53)$ \\
\hline Blinatumomab & $4(9)$ & $7(22)$ & $11(14)$ \\
\hline HCVD+ino & $21(46)$ & $5(16)$ & $26(33)$ \\
\hline
\end{tabular}

${ }^{a}$ Continuous variables are listed as median (range) and categorical variables as $\mathrm{n}(\%)$

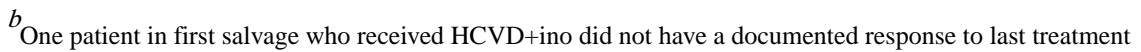

WBC, white blood cells; BM, bone marrow; LDH, lactate dehydrogenase; CNS, central nervous system; SCT, stem cell transplant; HCVD+ino; hyperfractionated cyclophosphamide, vincristine and dexamethasone plus inotuzumab 